\title{
动态扫描在 NR-PC 平板透镜目标探测成像中的应用
}

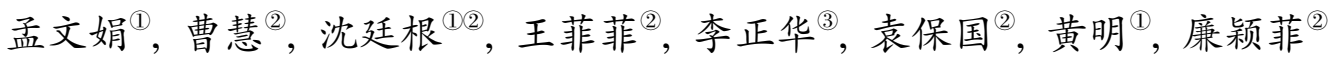 \\ (1) 江苏大学通信系, 镇江 212013; \\ (2) 江苏大学应用物理研究所, 镇江 212013; \\ (3) 镇江船艇学院通信系, 镇江 212013 \\ E-mail: mengujs2010@gmail.com \\ 2010-08-26 收稿, 2010-11-30 接受
}

\begin{abstract}
摘要 用时域有限差分法(FDTD)研究了基于等效负折射光子晶体(NR-PC)平板透镜的动态 扫描系统在目标探测成像中的应用. 通过对负折射光子晶体(NR-PC)平板透镜的仿真和研究 得到: 由于微禁带和共振激发效应, 在共振频率 $0.3068(a / \lambda)$ 处出现很强的光波透射峰, 从点 源发出的光波在经由 NR-PC 平板透镜聚焦于目标后，在该处将会产生极强的后向散射波，使 得后向散射波的再聚焦横向扫描分辨率和图像分辨率有了极大的提高. 另外, 通过将非动态 扫描方案与动态扫描方案进行对比, 可以发现动态扫描方案能够获得较好的再聚焦分辨率, 具有一定的优越性. 为将理想化的 LHM 平板透镜探测成像系统转化为可实现的 NR-PC 平板 透镜系统提供了重要依据，从而对优化探测成像系统的性能、实现小目标的探测及成像具有 十分重要的意义.
\end{abstract}

关键词

等效负折射

动态扫描

散射信号

再聚焦分辨率
负折射率介质材料最早是由 Veselago 提出的 ${ }^{[1]}$. 之后, Smith 等人通过微波实验对该材料的独特的电 磁特性进行了更详尽的论证 ${ }^{[2]}$. 在他们的带动下, 众 多相关领域的学者逐渐将研究焦点集中在探索和研 究其更多新奇的电磁特性上 ${ }^{[3,4]}$. 由于负折射率介质 材料的介电常数 $(\varepsilon)$ 和磁导率 $(\mu)$ 都小于 0 , 当电磁波 在这种介质材料中传播时, 电场、磁场和波矢遵从 左手法则，因而，该材料又被称为左手材料(LHM).

LHM 在近场目标探测及成像上得到了人们的特 别关注, 具有平坦表面的负折射率 LHM 平板透镜亦 被提出用作聚焦透镜 ${ }^{[5]}$. 理论分析及数值模拟 ${ }^{[6]}$ 表明 由 LHM 制作而成的无损耗的所谓完美透镜将获得突 破衍射极限的聚焦分辨率, 且较高的聚焦分辨率通 常会产生较高的图像分辨率 ${ }^{[7]}$.

诚然, LHM 平板透镜在近场目标探测及成像方 面取得了显著的成绩 ${ }^{[7]}$, 但遗憾的是, 自然界中迄今 尚未发现介电常数和磁导率同时为负值的天然 LHM
材料. 为此, Notomi 曾深人研究和阐述通过不同的方 法在二维光子晶体 $(\mathrm{PC})$ 模型中获得介质的等效负折 射率 ${ }^{[8]}$. 在这种光子晶体中, 在接近禁带附近的等频 率线会近似为圆形 ${ }^{[9]}$, 这意味着在这些频率范围内, 光在光子晶体中的传播类似于在普通各向同性介质 中传播. 这样, 我们可以用等效折射率 $n_{\mathrm{eff}}$ 来描述光 在此晶体中的传播, 由此产生等效负折射光子晶体 (NR-PC)概念. NR-PC 平板透镜的成像遵循几何光学 特性 ${ }^{[10,11]}$, 因而可以将其应用于光波目标探测及成 像系统中.

本文中，我们考虑二维光子晶体是由在 $\mathrm{GaAs}$ 介 质基底刻蚀上三角排列的圆柱型空气孔构成, $Z$ 方向 7 行, $X$ 方向取 30 列, 如图 1(a)所示. 其中, GaAs 介 质基底的相对介电常数是 $\varepsilon=12.96$, 空气孔的半径 是 $0.4 a$ (其中 $a$ 是二维光子晶体的晶格常数). 对于 $\mathrm{TM}$ 模, 该结构的光子晶体的等效折射率 $n_{\mathrm{eff}}$ 是随归 一化频率 $\omega$ (单位为 $a / \lambda$ ) 变化的 ${ }^{[8]}$, 如图 1(b)所示. 从 


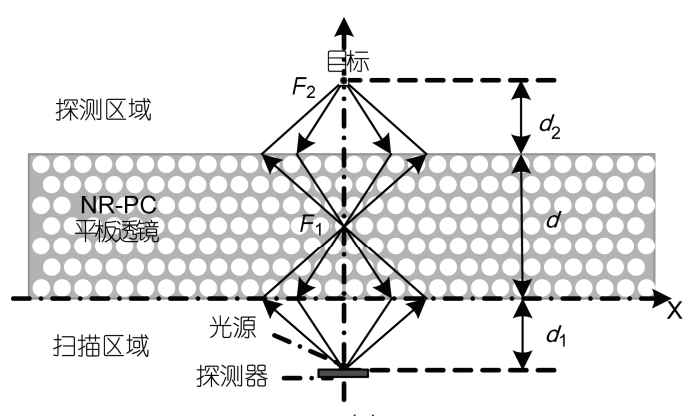

(a)

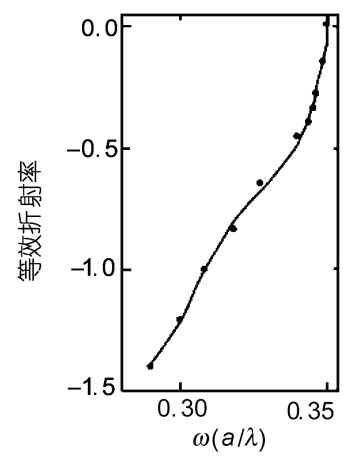

(b)

图 1 (a) NR-PC 平板透镜成像及目标探测示意图; (b) 对于 TM 模, 光子晶体的等效折射率 $n_{\mathrm{eff}}$ 随归一化频率 $\omega$ 变化曲线图

中可以看到, 当 $0.3 \leqslant \omega \leqslant 0.35$ 时, $n_{\mathrm{eff}} \leqslant 0$, 且 $n_{\mathrm{eff}} \approx-1$ 时 所对应的归一化频率 $\omega \approx 0.3068(a / \lambda)$.

与 LHM 平板透镜应用于目标探测成像的原理 ${ }^{[5]}$ 类似，图 1(a)所示的 NR-PC 平板透镜目标探测及成 像原理可叙述如下: 从点光源发出的光波先在 NR-PC 平板透镜内部焦点 $F_{1}$ 处聚焦, 之后在 NR-PC 平板透镜外部焦点 $F_{2}$ 处聚焦, 如果刚好在 $F_{2}$ 处有一 个目标, 该目标将对聚焦的光波产生后向散射作用, 经后向散射的光波将被 NR-PC 平板透镜再聚焦于光 源附近. 通过调整点源与平板透镜间的距离或沿与 平板表面平行的方向移动点源, 可以很方便地调整 源在 NR-PC 平板透镜另一侧的聚焦点的位置, 使其 灵活地聚焦于目标处. 因此, 来自目标的散射信号 得到了显著的增强. 此时, 光源附近的每个接收点 处记录的总的光波实际上包含 3 部分: 点源发出的 波、进出 NR-PC 平板透镜表面反射的波(对于等效负 折射率 $n_{\mathrm{eff}} \neq-1$ 的透镜)和目标后向散射再聚焦后的 波. 为了获得后向散射波(散射信号), 我们用有目标 位于 $F_{2}$ 处时记录的场强大小减去无目标时的场强大 小. 因此, 只需要简单地将在不同接收位置所获得
的散射信号电平分布显示出来, 即可完成对目标的 探测与成像 ${ }^{[6]}$.

本文研究了基于 NR-PC 平板透镜的动态扫描系 统在目标探测成像中的重要作用. 首先, 我们用二 维有限时域差分法研究并发现 NR-PC 平板透镜在共 振频率 $0.3068(a / \lambda)$ 处出现很强的光波透射峰, 导致 目标处产生极强的后向散射波, 使得后向散射波的 再聚焦横向扫描分辨率和图像分辨率有了极大的提 高, 从而进一步优化了扫描-聚焦成像系统的性能. 之后, 针对图 1(a)所讨论的 NR-PC 平板透镜的设计 及计算模型进行了数值分析, 并利用 NR-PC 平板透 镜对目标进行动态扫描探测. 最后, 将上述动态扫 描方案与非动态扫描方案进行对比, 从而进一步证 明基于 NR-PC 平板透镜的动态扫描系统在目标探测 成像中可以发挥重要的作用.

\section{NR-PC 平板透镜的设计及计算模型}

电磁波(光场)在光子晶体中的 Maxwell 方程满足

$$
\begin{gathered}
\frac{\partial \vec{H}}{\partial t}=-\frac{1}{\mu_{0} \mu(r)} \nabla \times \vec{E}, \\
\frac{\partial \vec{E}}{\partial t}=\frac{1}{\varepsilon_{0} \varepsilon(r)} \nabla \times \vec{H},
\end{gathered}
$$

式中 $\nabla=\frac{\partial}{\partial x} \vec{i}+\frac{\partial}{\partial y} \vec{j}+\frac{\partial}{\partial z} \vec{k}$ 是哈密顿算符, $\vec{E}$ 与 $\vec{H}$ 分 别是光子晶体中电磁波的电场与磁场矢量, 空间坐 标函数 $\varepsilon(r)$ 与 $\mu(r)=1$ 分别为光子晶体介质的介电常数 及相对磁导率.

本文的研究是借助电磁场数值计算方法-时域有 限差分法(FDTD $)^{[12]}$ 实现的. 该方法的计算精度非常 高, 能很好地说明光子晶体的各种传输特性. 计算 中我们采用 TM 模, 通过求解上述麦克斯韦方程组, 将其在直角坐标系中展开成标量场分量的方程组, 然后用二阶精度的数值差商代替微商, 用 Yee 提出 的方法 ${ }^{[12]}$ 对上述微分方程进行时空离散化, 将连续 的空间和时间问题离散化, 即可得到标量场分量的 差分方程组. 之后, 通过编程和探测器仿真, 我们得 以进一步对 NR-PC 平板透镜在目标探测成像中的性 能进行研究. 在计算程序中, 我们使用的是在 $X, Z$ 方向采用完全匹配层(perfectly matched layer, PML) 的边界条件 ${ }^{[12]}$. 
值得注意的是, 光在 $n_{\mathrm{eff}}=-1$ 的光子晶体(PC)中 的传播方式完全不同于左手材料. 对于 $n=-1$ 的 LHM 来说, 空气与 LHM 之间的接触面上将不会发生反 射, 而对于 PC 而言, 即使 $n_{\text {eff }}=-1$, 在其与空气的接 触面上仍会发生多重反射和折射, 导致光在光子晶 体中的传播有极大的损耗, 其光能透射率 $T$ 较低. 因 而, 在优化扫描-聚焦成像系统的性能之前, 必须找 到有效提高光子晶体的光能透射率的途径.

针对这一问题, 我们已经做过相应的研究, 详 见 OPTIK 刊载论文 ${ }^{[13]}$. 当波源中心频率 $\omega_{\mathrm{p}} \approx 0.3068$ $(a / \lambda)$ 时, 在该 NR-PC 平板的归一化频率 $0.3068(a / \lambda)$ 处, 光子晶体的出射和人射平均能流的比值透射率 $T=4500$, 远大于 1 , 即光波透射率在波源中心频率为 $0.3068(a / \lambda)$ 处得到极大的提高. 我们认为, 这是由于 光子晶体的等效负折射结构的介质呈周期性分布, 当光子晶体等效折射率常数 $n_{\mathrm{eff}}=-1$ (它对应的归一化 频率 $\omega \approx 0.3068(a / \lambda)$ ) 时, 光波在该等效负折射光子 晶体界面及内部受强烈的布拉格散射的影响引起导 模, 进而产生微禁带效应, 同时, 光子隧道贯穿极大地 增强了共振激发效应 ${ }^{[14,15]}$, 使对于特定厚度的波导 内每个导模只能存在于较窄的频段内, 它使能量被 高度局域, 这是场的频域能量重新分配的结果, 进 而大大增强了它们的透射率, 导致它在对应的归一 化频率 $\omega \approx 0.3068(a / \lambda)$ 处形成窄的共振激发透射峰.

\section{2 关于 NR-PC 平板透镜后向散射波的横 向扫描再聚焦目标探测成像的讨论}

NR-PC 平板透镜探测成像系统的性能与分辨率 密切相关. 通过调控相应目标散射信号分辨率的大 小, 我们可以方便地研究和优化其性能. 特别是目 前, 对小目标进行探测成像的研究尚处于初级阶段, 急需高灵敏度的优质探测成像系统, 因此, 我们的 研究具有重要的意义.

下面的仿真中, 我们选取光源的中心频率为 $0.3068(a / \lambda)$, 将其放置在 $(-\lambda, 0)$ 处, 同时将厚度为 $d=2 \lambda$ 的 NR-PC 平板放置在 $0 \leqslant z \leqslant 2 \lambda$ 的区域, 并将边 长为 $L=1 / 3 \lambda$ 的正方形完美电导体(PEC) 目标放置于 焦点 $F_{2}$ 处. 为了获得来自该正方形目标的后向散射 波, 我们在 $z=-\lambda$ 处, 沿 $\boldsymbol{X}$ 轴方向以 $0.2 \mu \mathrm{m}$ 的步长同 步移动光源与探测器, 通过数值仿真得到计算区域 内的总光波场能量分布及由探测器记录的总光波场
强电平分布剖面图, 分别如图 2(a)及 2(b)所示.

不难发现, NR-PC 平板透镜遵循几何光学特性, 如图 2(a)中实线所示. 同时, 图 2(a)中沿 $z=-\lambda$ 处横向 显示的光波场能量分布与图 2(b)所示的由探测器记 录的总场强电平分布剖面图是有彼此对应的关系, 二者均表明: 聚焦场强的最大值出现在点光源附近.

正如前文所述，图 2 所示的是总光波分布，而 NR-PC 平板透镜对目标的探测成像作用主要体现在 目标的后向散射信号上. 因此, 我们将上述仿真中 设置的边长为 $L=1 / 3 \lambda$ 的正方形目标去掉, 在无目标 的情况下再次沿 $\boldsymbol{X}$ 轴移动光源与探测器进行动态扫 描. 仿真结束后, 同样记录下目标不存在时探测得 到的信号电平值, 并将其与目标存在时探测得到的 信号电平值对应相减, 从而得到相应于边长 $L=1 / 3 \lambda$ 的正方形 PEC 目标的散射信号电平值. 之后, 我们 进一步选取波源的中心频率为 $0.2068(a / \lambda)$, 在其余 条件不变的情况下进行相同的仿真，并将最终得到 的目标散射信号电平值与中心频率为 $0.3068(a / \lambda)$ 时 得到的数据进行对比, 得到相应的散射信号归一化 场强电平分布如图 3 所示.

在图 3 中, 通过测量散射信号场强电平分布剖 面图在最大值的 0.707 倍处的总宽度 ${ }^{[16]}$, 我们得知, 当波源的中心频率分别为 $0.3068(a / \lambda)$ 和 $0.2068(a / \lambda)$ 时，其相应的再聚焦分辨率分别为 $0.3718 \lambda$ 和 $1.59 \lambda$.

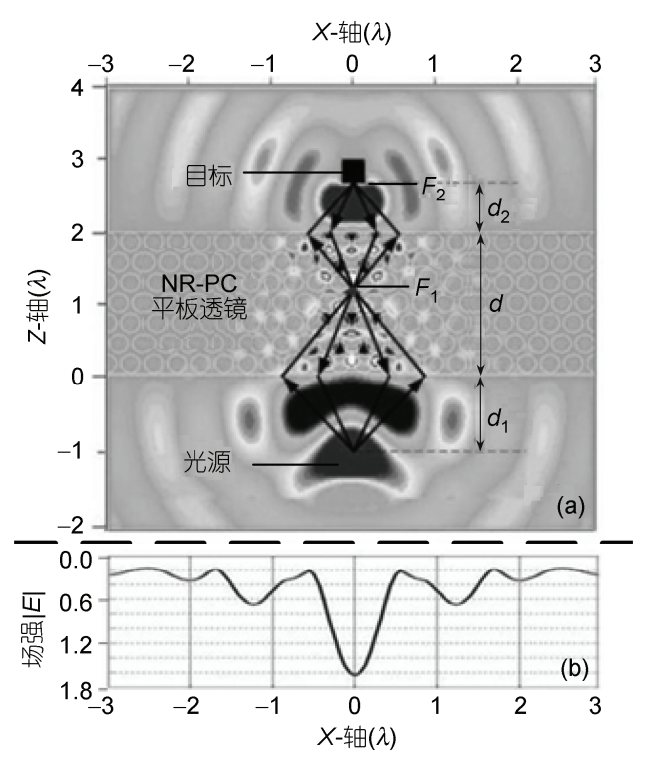

图 2 (a) 计算区域内的总光波场能量分布图; (b) 沿 $z=-\lambda$ 处, 横向记录的总光波场强电平分布剖面图 


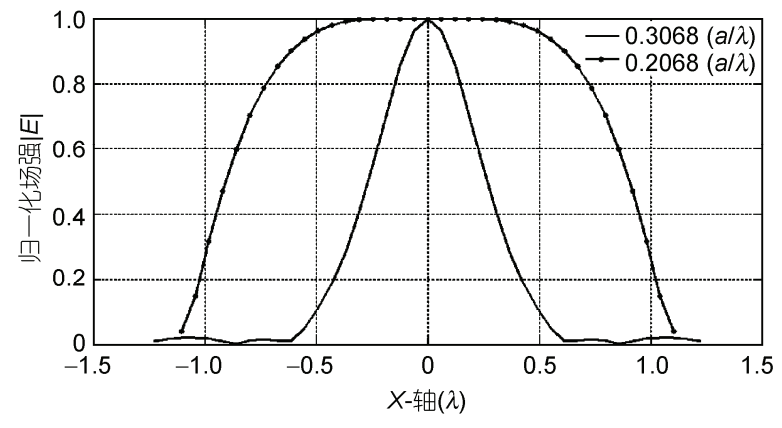

图 3 中心频率分别为 $0.3068(a / \lambda)$ 及 $0.2068(a / \lambda)$ 时对边长 $L=1 / 3 \lambda$ 的正方形目标进行探测得到的散射信号归一化场强 电平分布剖面图

由此可见, $0.3068(a / \lambda)$ 处聚焦横向分辨率远高于 $0.2068(a / \lambda)$ 处分辨率近 4 倍多, 我们认为, 这是由于 当选用中心频率为 $0.3068(a / \lambda)$ 的光源时, 在共振频 率 $0.3068(a / \lambda)$ 处会出现很强的光波透射峰, 导致目 标处产生极强的后向散射波, 使得后向散射波的再 聚焦横向扫描分辨率和图像分辨率得到极大的提高.

\section{3 基于 NR-PC 平板透镜的动态扫描方案 在目标探测成像中的重要作用}

在上述研究中, 我们采用的是动态扫描方案, 即要求将光源与探测器组合在一起同步移动. 为了 进一步说明该方案在基于 NR-PC 平板透镜的目标探 测成像中的优势, 我们将其与非动态扫描方案进行 对比. 该方案要求固定光源, 仅通过移动探测器来 实现对目标的探测成像.

以下仿真依然选用 $0.3068(a / \lambda)$ 作为光源的中心 频率. 在其他设置条件不变的前提下, 分别采用动 态扫描方案与非动态扫描方案来探测位于焦点 $F_{2}$ 处 的边长 $L=1 / 6 \lambda, 1 / 10 \lambda$ 及 $1 / 30 \lambda$ 的正方形 PEC 目标. 数 值分析后得到相应的散射信号归一化场强电平分布 剖面图, 如图 4 所示. 其中, 实线代表动态扫描方案, 虚线代表非动态扫描方案.

根据图 4 与表一可知, 与非动态扫描方案相比, 动态扫描方案在目标探测成像中可获得更好的再聚 焦分辨率. 当对边长为 $L=1 / 6 \lambda, 1 / 10 \lambda, 1 / 30 \lambda$ 的正方 形 PEC 目标进行探测时, 动态扫描方案下得到的相 应的再聚焦分辨率较非动态扫描方案分别提高了 $0.06,0.24,0.25$ 倍. 也就是说, 随着目标的减小, 动 态扫描方案在目标探测成像中的优势亦发明显. 同

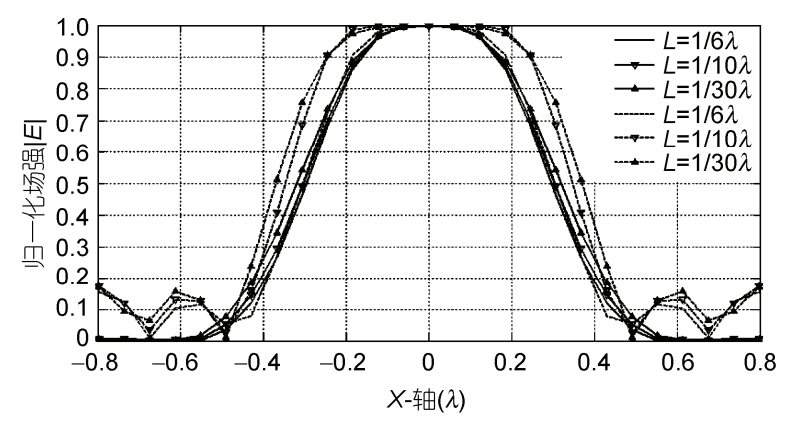

图 4 动态及非动态扫描方案下得到的正方形目标的散射信 号归一化场强电平分布剖面图

表 1 动态及非动态扫描方案下针对方形目标进行探测后 得到的分辨率对比

\begin{tabular}{cccc}
\hline 正方形目标边长 & $L=1 / 6 \lambda$ & $L=1 / 10 \lambda$ & $L=1 / 30 \lambda$ \\
\hline 动态扫描再聚焦分辨率 & $0.4736 \lambda$ & $0.4868 \lambda$ & $0.51 \lambda$ \\
非动态扫描再聚焦分辨率 & $0.5004 \lambda$ & $0.6014 \lambda$ & $0.6388 \lambda$ \\
\hline
\end{tabular}

时, 无论是选用动态亦或非动态扫描方案, 散射信 号再聚焦分辨率都是随着目标尺寸的增大而不断提 高的, 这完全符合一般的物理规律.

为了进一步研究动态扫描方案在基于 NR-PC 平 板透镜的目标探测成像中的重要作用, 我们对探测 目标的形状稍作调整, 即分别选用直径 $D=1 / 6 \lambda$, $1 / 10 \lambda, 1 / 30 \lambda$ 的圆形 PEC 目标进行探测, 并将相应的 再聚焦分辨率数据与方形目标下得到的数据进行对 比, 如图 5 及表 2 所示.

在文献[7]中提到, 利用 LHM 平板透镜对直径为 $D=1 / 6 \lambda$ 的圆形 PEC 目标进行动态扫描, 可得到再聚 焦分辨率为 $0.257 \lambda$. 而在本文中, 当使用 NR-PC 平 板透镜时, 相应的动态扫描再聚焦分辨率为 $0.2564 \lambda$,

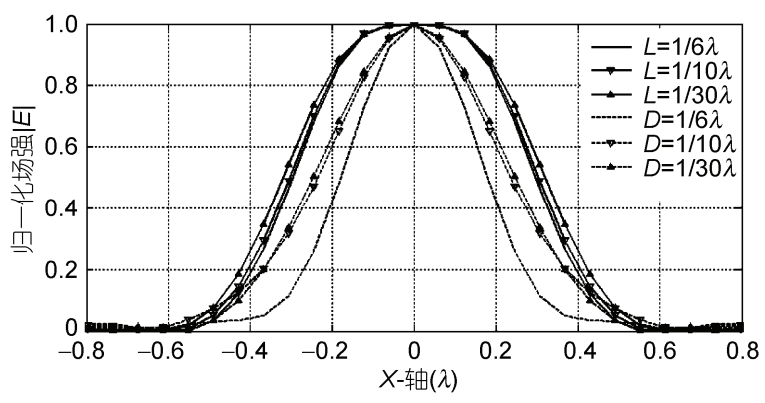

图 5 动态扫描方案下得到的正方形及圆形目标的散射信号 归一化场强电平分布剖面图 
表 2 动态扫描方案下针对方形及圆形目标进行探测后得 到的分辨率对比

\begin{tabular}{lll}
\hline & \multicolumn{1}{c}{ 目标大小 } & \multicolumn{1}{c}{ 分辨率 } \\
\hline & 边长 $L=1 / 6 \lambda$ & $0.4736 \lambda$ \\
正方形目标 & 边长 $L=1 / 10 \lambda$ & $0.4868 \lambda$ \\
& 边长 $L=1 / 30 \lambda$ & $0.51 \lambda$ \\
& 直径 $D=1 / 6 \lambda$ & $0.2564 \lambda$ \\
圆形目标 & 直径 $D=1 / 10 \lambda$ & $0.3286 \lambda$ \\
& 直径 $D=1 / 30 \lambda$ & $0.3518 \lambda$ \\
\hline
\end{tabular}

二者近似相等. 可见, NR-PC 平板透镜的动态扫描成 像系统与 LHM 平板透镜的动态扫描成像系统的成像 精度近似相等. 同时, 结合图 5 与表 2 可知, 对圆形 目标进行探测得到的散射信号再聚焦分辨率普遍优 于方形目标.

\section{4 结论}

本文主要利用二维时域有限差分法, 结合动态 扫描方案对 NR-PC 平板透镜进行仿真和研究. 由于 微禁带和共振激发效应, 光波在经过 NR-PC 平板时,
在共振频率 $0.3068(a / \lambda)$ 处会出现很强的光波透射峰. 同时, NR-PC 平板透镜的聚焦放大特性和修逝波在 该 NR-PC 平板透镜中的指数放大作用 ${ }^{[5]}$, 使得从中 心频率为 $0.3068(a / \lambda)$ 的点源发出的光波经 NR-PC 平 板透镜聚焦于目标后，在目标处产生极强的后向散 射波，进而使目标后向散射波的再聚焦横向扫描分 辨率和图像分辨率有了极大的提高. 通过对动态扫 描方案与非动态扫描方案的性能分析, 可以发现动 态扫描方案在提高系统的图像分辨率上具有较为明 显的优越性. 同时, 通过对直径 $D=1 / 6 \lambda$ 的圆形目标 进行动态扫描探测, 可知 NR-PC 平板透镜的目标探 测成像系统与 LHM 平板透镜的成像精度近似相等. 进一步研究发现, 对圆形形目标进行探测得到的散 射信号再聚焦分辨率普遍优于方形目标.

本文的研究为将理想化的 LHM 平板透镜目标 探测成像系统转为可实现的 NR-PC 平板透镜成像 系统提供了重要依据, 这对优化目标探测成像系 统的性能及实现小目标的探测及成像具有十分重 要的意义.

\section{参考文献}

1 Veselago V G. The electrodynamics of substances with simultaneously negative values of $\varepsilon$ and $\mu$. Sov Phys Usp, 1968, 10: 509-514

2 Shelby R A, Smith D R, Schultz S. Experimental verification of a negative index of refraction. Science, 2001, 292: 77-79

3 Zhao Q, Zhao X P, Kang L, et al. Reflection and phase of left-handed metamaterials at microwave frequencies. Chinese Sci Bull, 2005, 50: 395-398

4 Cao Y J, Wen G J, Wu K M, et al. A novel approach to design microwave medium of negative refractive index and simulation verification. Chinese Sci Bull, 2007, 52: 433-439

5 Pendry J B. Negative refraction makes a perfect lens. Phys Rev Lett, 2000, 85: 3966-3969

6 Rao X S, Ong C K. Subwavelength imaging by a left-handed material superlens. Phys Rev E, 2003, 68: 067601-067603

7 Wang G, Fang J R, Dong X T. Resolution of Near-field microwave target detection and imaging by using flat LHM lens. IEEE Trans Antennas Propog, 2007, 55: 3534-3541

8 Notomi M. Theory of light propagation in strongly modulated photonic crystals: Refractionlike behavior in the vicinity of the photonic band gap. Phys Rev B, 2000, 62: 10696-10706

9 Guven K, Aydin K, Alici K B, et al. Spectral negative refraction and focusing analysis of a two-dimensional left-handed photonic crystal lens. Phys Rev B, 2004, 70: 205125-205130

10 Fang Y T, Sun H J, Shen T G. New evidences of negative refraction in photonic crystals. Opt Mater, 2006, 28: 1156-1159

11 Fang Y T, Shen T G. Multi-imaging by photonic crystal slab using negative refraction. Chin Phys Lett, 2005, 22: 949-951

12 Yee K S. Numerical solution of initial boundary value problems involving Maxwell's equations in isotropic media. IEEE Trans Antennas Propog, 1966, 14: 302-308

13 Li Z H, Xue Y L, Deng Z Q, et al. Study on optical switching effect of photonic crystals with negative effective index of refraction. Optik, 2009, 120: 605-609

14 Sharon A, Rosenblatt D, Friesem A A. Narrow spectral bandwidths with grating waveguide structures. Appl Phys Lett, 1996, 69: 4154-4156

15 Shadrivov I V, Sukhorukov A A, Kivshar Y S, et al. Nonlinear surface waves in left-handed materials. Phys Rev E, 2004, 69: 016617-016619

16 Grbic A, Eleftheriades G V. Overcoming the diffraction limit with a planar left-handed transmission-line lens. Phys Rev Lett, 2004, 92: 117403-117406 